\title{
Inhibition of nm23-H1 gene expression in chronic myelogenous leukemia cells
}

\author{
ZHENSHENG DAI $^{1 *}$, WEIZHONG XIAO ${ }^{2 *}$ and YUELING JIN ${ }^{3}$ \\ Departments of ${ }^{1}$ Hematology and ${ }^{2}$ Neurology, Shanghai Pudong Hospital, Affiliated to Fudan University, Shanghai 201399; \\ ${ }^{3}$ Department of Pathology, Shanghai Health Vocational Technical College, Xuhui, Shanghai 200237, P.R. China
}

Received February 17, 2013; Accepted July 19, 2013

DOI: $10.3892 / 01.2013 .1500$

\begin{abstract}
For solid tumors of a malignant origin, the expression of the nm23-H1 gene is a positive prognostic factor. However, for chronic myeloid leukemia (CML), the prognostic role of $\mathrm{nm} 23-\mathrm{H} 1$ gene expression is unknown. The present study investigated the impact of $\mathrm{nm} 23-\mathrm{H} 1$ gene expression on the proliferation and migration of the CML K562 cell line to elucidate the association between $\mathrm{nm} 23-\mathrm{H} 1$ gene expression and CML cell survival. An RNAi lipo-recombinant plasmid of the nm23-H1 gene (pGCsi-nm23-H1) was constructed and transfected into the K562 cells. RT-PCR and western blotting were used to detect nm23-H1 mRNA and protein expression, respectively. The anchorage-independent growth ability of the transfected cells was observed in soft agar culture and the ability of the K562 cells to migrate was determined using a Transwell assay. Following the successful construction and transfection of the pGCsi-nm23-H1 plasmid into the K562 cells, nm23-H1 mRNA and protein expression levels were significantly lower compared with the control group. The stably-transfected pGCsi-nm23-H1 K562 cells exhibited a markedly increased ability to form colonies and the number and sizes of the colonies were significantly increased compared with those of the control. In vitro, the cells migrated through a Matrigel-coated membrane during incubation for $20 \mathrm{~h}$. The Transwell assay revealed that the quantitative number of pGCsi-nm23-H1 K562 cells that migrated into the lower compartment of the invasion chamber was markedly increased compared with the control. In conclusion, nm23-H1 gene expression may inhibit K562 cell proliferation and migration. $\mathrm{nm} 23-\mathrm{H} 1$ may be a
\end{abstract}

Correspondence to: Dr Zhensheng Dai, Department of Hematology, Shanghai Pudong Hospital, Affiliated to Fudan University, 2008 Gongwei Road, Huinan, Shanghai 201399, P.R. China

E-mail: zhenshengdai@yahoo.com.cn

${ }^{*}$ Contributed equally

Key words: nm23-H1, chronic myelogenous leukemia, RNAi, migration cancer suppressor gene and play a significant role in inhibiting the survival of CML cells.

\section{Introduction}

Our knowledge of the pathogenesis of leukemia, including an understanding of its molecular mechanisms, has progressed as numerous studies have been undertaken with regard to the various aspects of gene therapy. The prognosis of patients with leukemia is closely associated with the invasion and metastasis of the malignant cells. The nm23-H1 gene is a tumor metastasis suppressor gene. The effect of the gene on the prognosis and tumor metastasis have been described in studies of solid tumors, including those of gall bladder $(1,2)$ liver $(3,4)$ and gastric (5) cancer.

For malignant tumors of the blood system, the expression of the $\mathrm{nm} 23-\mathrm{H} 1$ gene is a poor prognostic factor $(6,7)$. Magyarosy et al (8) studied nm23-H1 expression in acute lymphoblastic leukemia and revealed that the expression of $\mathrm{nm} 23-\mathrm{H} 1$ in low-differentiated cells was higher than that in relatively well-differentiated cells. Therefore, the nm23-H1 gene was considered to be a prognostic marker for a variety of cancers of the blood system. The K562 cell line originates from chronic myeloid leukemia (CML). Currently, studies on the $\mathrm{nm} 23-\mathrm{H} 1$ gene in CML are rare. Therefore, in the present study, the RNAi technique was used to inhibit nm23-H1 gene expression in the K562 cell line to investigate the affects of $\mathrm{nm} 23-\mathrm{H} 1$ gene expression on the proliferation and migration of the K562 cells and to further clarify its correlation with prognosis for the molecular targeted treatment of CML.

\section{Materials and methods}

Cell lines. K562 cells were obtained from the Shanghai Institute of Cell Biology, Chinese Academy of Sciences (Shanghai, China). The cells were cultured at $37^{\circ} \mathrm{C}$ in humidified 5\% $\mathrm{CO}_{2}$ in RPMI-1640 medium (Sigma, St. Louis, MO, USA), supplemented with $10 \%$ fetal bovine serum and 100 units $/ \mathrm{ml}$ penicillin and streptomycin.

Short hairpin RNA (shRNA) preparation and plasmid construction. Two pairs of shRNA sequences were designed, one according to the nm23-H1 sequence in GenBank (D1734) and the other sequence with no homology to the human sequence, 
Table I. List of primer sequences and amplification conditions used in the PCR.

\begin{tabular}{|c|c|c|c|}
\hline Gene & Primer sequences $\left(5^{\prime}-3^{\prime}\right)$ & PCR conditions & Product size (bp) \\
\hline $\mathrm{nm} 23-\mathrm{H} 1$ & $\begin{array}{l}\text { 5'-TTAATCAGATGGTCGGGGAT-3' } \\
\text { 5'-GATCTATGAATGACAGGAGG-3' }\end{array}$ & $\begin{array}{l}94^{\circ} \mathrm{C}, 30 \mathrm{sec} ; 56^{\circ} \mathrm{C}, 30 \mathrm{sec}, \\
72^{\circ} \mathrm{C}, 30 \mathrm{sec} ; 32 \text { cycles }\end{array}$ & 186 \\
\hline$\beta$-actin & $\begin{array}{l}\text { 5'-CGTGGCCTTAGCTGTGCT-3' } \\
\text { 5'-TGTGCATAAAGTGTAAGTGTATAAGCA-3' }\end{array}$ & $\begin{array}{l}94^{\circ} \mathrm{C}, 30 \mathrm{sec} ; 54^{\circ} \mathrm{C}, 30 \mathrm{sec} \\
72^{\circ} \mathrm{C}, 30 \mathrm{sec} ; 32 \text { cycles }\end{array}$ & 457 \\
\hline
\end{tabular}

which was used as a control. Each pair contained a unique 19-nt double-stranded sequence that was separated by a loop of 9-nt sequences (ttcaagaga). The oligonucleotide sequences of siRNA contained a BamHI and HindIII site. Subsequent to the purification and restriction digestion, the oligonucleotides were ligated into the pGCsi plasmid (GeneChem Inc., Shanghai, China) with the polymerase III U6 promoter. The nm23-H1 recombinant plasmid was confirmed by sequencing and named pGCsi-nm23-H1.

RNA extraction and semi-quantitative RT-PCR. Total RNA extraction was performed using TRIzol reagent (Takara, Shiga, Japan). The reverse transcription reaction was performed using $2 \mu \mathrm{g}$ total RNA with a first strand cDNA kit (Takara), according to the manufacturer's instructions. PCR was performed in a $25-\mu 1$ reaction volume containing $2 \mu 1 \mathrm{cDNA}$ template, 10X buffer, $0.15 \mathrm{mM}$ dNTP, $0.1 \mathrm{mM}$ of each primer and 0.5 U Ex Taq Hot Start Version (Takara). The primers and the amplification conditions that were used in the PCR are listed in Table I. The final products were identified in $1.7 \%$ agarose gel and stained with ethidium bromide.

Transfection assay. To generate the nm23-H1 siRNA-transfected K562 cells, $3 \mu \mathrm{g}$ plasmid DNA was transfected into $1 \times 10^{5}$ cells in a $60-\mathrm{mm}$ dish using lipofectamine 2000 (Invitrogen, Carlsbad, CA, USA), according to the manufacturer's instructions. The transfected cells were selected in a medium containing $400 \mu \mathrm{g} / \mathrm{ml} \mathrm{G} 418$ (Geneticin; Invitrogen) and the stable nm23-H1 siRNA-transfected cells were named pGCsi-nm23-H1 K562 cells. The control K562 cells were transfected with liposome and named the liposome K562 cells.

Western blotting. Each group of cells was washed twice with phosphate-buffered saline (PBS), lysed for $10 \mathrm{~min}$ in hot water and centrifuged at 20,000 x g for $10 \mathrm{~min}$. Total proteins $(10 \mu \mathrm{l})$ were separated by $5 \%$ sodium dodecyl sulfate polyacrylamide gel electrophoresis (SDS-PAGE) and transferred onto a polyvinylidene fluoride (PVDF) membrane. Subsequent to being immersed in $10 \mathrm{ml} 5 \%$ skimmed milk in TBST solution for $1 \mathrm{~h}$, the membrane was incubated with primary and secondary antibodies. Human monoclonal anti-nm23-H1 (1:300; BD Biosciences Pharmingen, San Diego, CA, USA) and $\beta$-actin (1:500; Invitrogen) antibodies were used as the primary antibodies. Bovine anti-mouse $\operatorname{IgG}$ (1:2500; Santa Cruz Biotechnologies, Santa Cruz, CA) was used as the secondary antibody. Finally, images of the results were captured with an enhanced chemiluminescence (ECL) substrate.
MTT assay. For the cell proliferation assays, each group of cells was plated in triplicate in 96-well plates at a density of $1 \times 10^{4}$ cells/well and grown for 1, 2,3, 4, 5, 6 and 7 days, respectively. A total of $20 \mu \mathrm{l} 5 \mathrm{mg} / \mathrm{ml}$ MTT was added. Following a 4-h incubation period, the number of metabolically active cells was quantified.

Colony formation assay. The cells $\left(1 \times 10^{3}\right)$ were seeded into 6 -well plates with $2 \mathrm{ml}$ culture medium. Subsequent to a two-week incubation period in RPMI-1640 medium supplemented with $10 \%$ fetal bovine serum at $37^{\circ} \mathrm{C}$ and $5 \% \mathrm{CO}_{2}$, the colonies were washed twice with PBS, stained with Giemsa, counted, visualized microscopically and had their images captured.

Transwell assay. For the migration assays, the pGCsil-nm23-H1 K562 and control liposome K562 cells were cultured in RPMI-1640 medium supplemented with $10 \%$ fetal bovine serum at $37^{\circ} \mathrm{C}$ and $5 \% \mathrm{CO}_{2}$. When the cells had grown to $80 \%$ confluence, they were incubated for $24 \mathrm{~h}$ in medium without fetal bovine serum. The cell culture supernatants were collected and preserved at $-20^{\circ} \mathrm{C}$ for further use as epidermal growth factor (EGF). The undersides of the Transwell chamber membranes (BD Biosciences Pharmingen) were coated with $250 \mu$ l Matrigel gels mixed with $250 \mu$ l RPMI-1640 medium. Each group contained $1 \times 10^{5}$ cells and was seeded on the Transwell chamber. Following this, $800 \mu 1$ NIH3T3 EGF that was prepared previously was added to the 6 -well plates. Following $24 \mathrm{~h}$, the Matrigel gel on the upper sides of the membranes was removed using cotton swabs. The Transwell chamber membranes were fixed in $95 \%$ ethanol for $15 \mathrm{~min}$. The cells that had migrated to the undersides of the membranes were stained with hematoxylin and eosin (HE) and counted by microscopy (x200). The results were determined by averaging the cell counts in five fields.

Statistics. The data were analyzed using the SPSS software program (v 11.0; SPSS, Inc., Chicago, IL, USA). Non-parametric tests were performed using independent samples. The mean values were compared by a one-way ANOVA. $\mathrm{P}<0.05$ was considered to indicate a statistically significant difference.

\section{Results}

Transfection assay. Following the transfection with the pGCsi-nm23-H1 plasmids, a change was observed in the morphology of the cells, and green fluorescence in the nucleus was visualized by fluorescence microscope. Following $48 \mathrm{~h}$, the efficiency of the plasmid transfection was calculated. Plasmid 


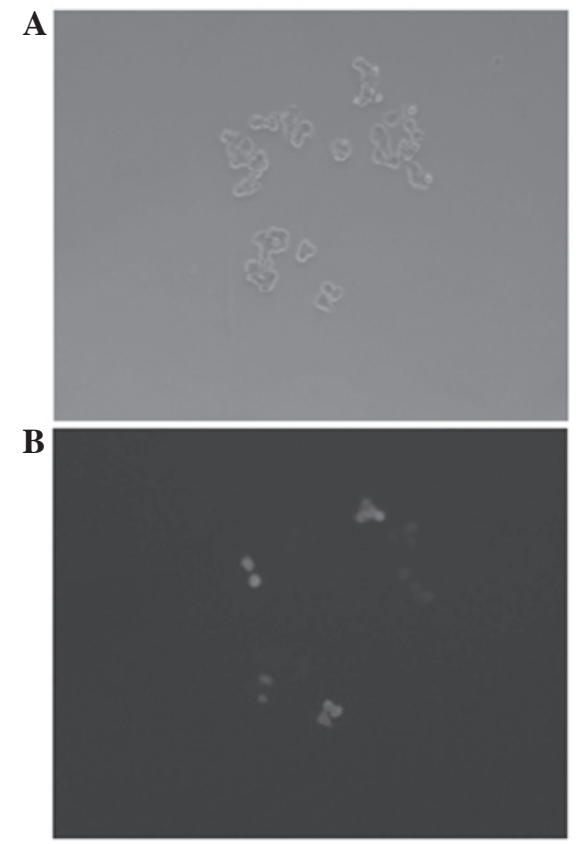

Figure 1. (A) Typical microscope image of pGCsi-nm23-H1 K562 cells. Slight changes in cell shape were observed. (B) Typical fluorescence microscope image of pGCsi-nm23-H1 562 cells. Green fluorescence is observed in the nucleus, which indicates that pGCsi-nm23-H1 has been transducted into the K562 cells successfully. (A and B) Magnification, x40.

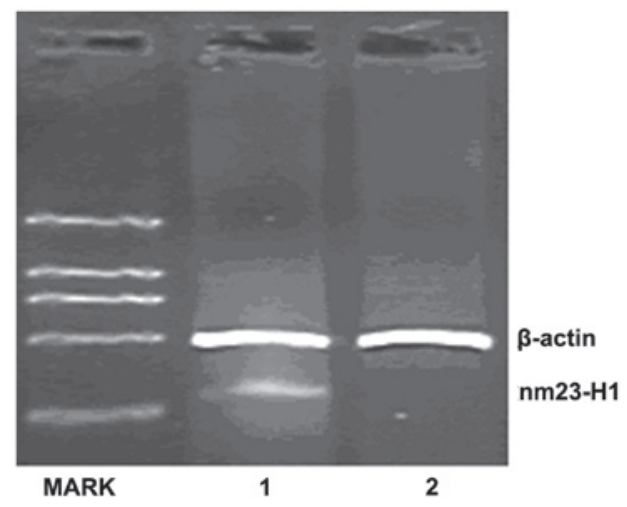

Figure 2.nm23-H1 mRNA expression. 1, liposome group; 2, pGCsi-nm23-H1 group. Inhibition of $\mathrm{nm} 23-\mathrm{H} 1$ expression in the K562 cell line. Semiquantitative PCR analysis was performed using total RNA extracted from the control and pGCsi-nm23-H1 K562 cells. $\beta$-actin was analyzed as a positive control. The expression of $\mathrm{nm} 23-\mathrm{H} 1 \mathrm{mRNA}$ was decreased in the pGCsi-nm23-H1 K562 cells. The sizes of nm23-H1 and $\beta$-actin were 252 bp and $457 \mathrm{bp}$, respectively.

transfection efficiency $=$ number of fluorescent cells per high power field (HPF) / number of cells in the same field x 100 (3). The transfection efficiency of the pGCsi-nm23-H1-transfected K562 cells was $\sim 40 \%$. (Fig. 1)

Inhibition of nm23-H1 gene expression by shRNA expression vectors. The knockdown efficiencies of nm23-H1-specific shRNA in the K562 cells were analyzed using semiquantitative PCR and western blotting. The relative nm23-H1 mRNA levels were normalized by internal control $\beta$-actin and the western blot assay for $\mathrm{nm} 23-\mathrm{H} 1$ protein expression was normalized by $\beta$-tubulin. Following transfection, the mRNA

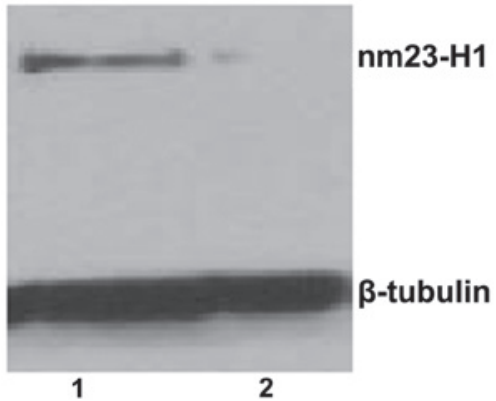

Figure 3. Western blot analysis of nm23-H1 expression. 1, liposome group; 2, pGCsi-nm23-H1 group. Bands of $40 \mathrm{kDa}$ represent signals of $\beta$-tubulin and bands of $17 \mathrm{kDa}$ represent signals of the $\mathrm{nm} 23-\mathrm{H} 1$ protein. Compared with the control group, a weaker expression of nm23-H1 was detected in the pGCsi-nm23-H1 K562 cells.

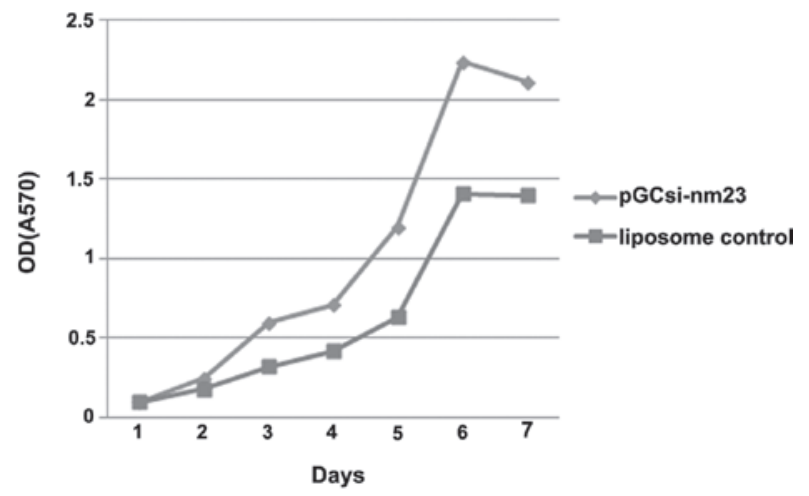

Figure 4. Cell proliferation was assessed by MTT assay. Cells were seeded in 96-well plates, grown for 1, 2, 3, 4, 5, 6 and 7 days and incubated with $20 \mu \mathrm{l}$ $5 \mathrm{mg} / \mathrm{ml}$ MTT for $4 \mathrm{~h}$. The quantification of the metabolic activity in the cells that were transfected with pGCsi-nm23-H1 was significantly higher than the cells of the liposome control groups, particularly after 4 days.

and protein expression levels of $\mathrm{nm} 23-\mathrm{H} 1$ were reduced in the pGCsi-nm23-H1 K562 cells. (Figs. 2 and 3)

MTT assay. For the cell proliferation assays on the inhibition of nm23-H1 gene expression by the shRNA expression vectors, the number of metabolically active cells was quantified. The quantification of the metabolic activity of the cells that were transfected with pGCsil-nm23-H1 was significantly higher than that in the cells of the control groups, particularly following 4 days of transfection. (Fig. 4)

nm23-H1-specific shRNAs induce cell forming colonies. To evaluate the tumor suppression function of $\mathrm{nm} 23-\mathrm{H} 1$ in CML, the anchorage-independent growth abilities of the pGCsi-nm23-H1 K562 and liposome control K562 cells were compared in soft agar culture. The stably-transfected pGCsi-nm23-H1-siRNA K562 cells exhibited a dramatically increased ability to form colonies on soft agar. The number and sizes of the colonies that were formed by the pGCsi-nm23-H1 K562 cells were significantly increased compared with those that were formed by the liposome control group (Fig. 5).

nm23-H1-specific shRNAs induce cell migration in vitro. To analyze whether the $\mathrm{nm} 23-\mathrm{H} 1$ gene was involved in the migration of the K562 cells, the effect of the invasiveness of 

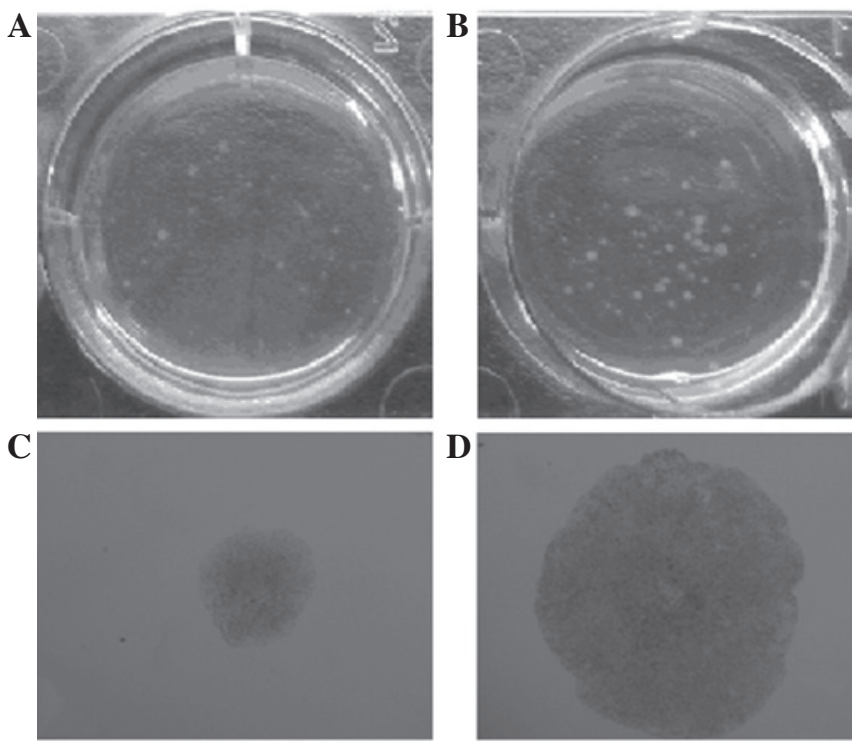

Figure 5. Inhibition of colony formation in K562 cells transfected with the nm23-H1 siRNA gene. Cells $\left(1 \times 10^{3}\right)$ were seeded in 6-well plates and cultured for 2 weeks. The colonies were stained with Giemsa, counted and had their images captured. A significant increase in the colony number and volume was observed in the (B) nm23-H1 siRNA-transfected K562 cells compared with the (A) liposome K562 cells. The number of colonies that were formed were taken from three independent experiments. In the pGCsi-nm23-H1 group, the mean \pm SD was $12.14 \pm 3.51$, while in the liposome control group this value was $4.32 \pm 0.95$, as visualized by microscopy $(\mathrm{x} 40)$. The size of the colonies formed by the (D) pGCsi-nm23-H1 K562 cells were significantly increased compared with the (C) liposome control group. SD, standard deviation.

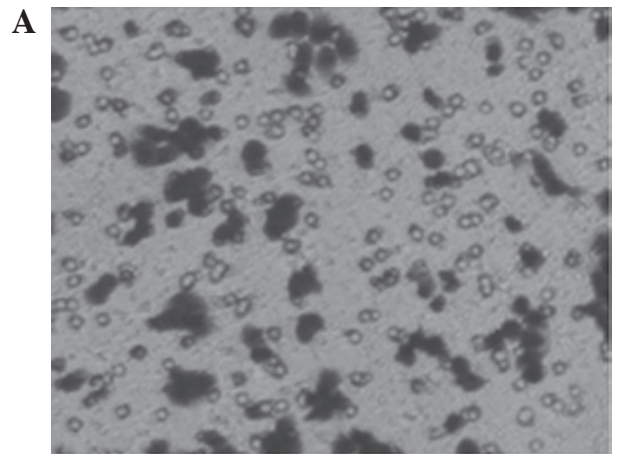

B

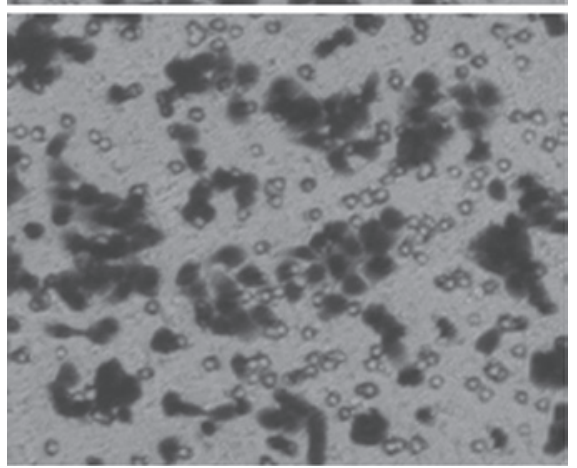

Figure 6. Detection of K562 invasiveness in vitro by the repression of $\mathrm{nm} 23-\mathrm{H} 1$ expression. The ability of pGCsi-nm23-H1 K562 cells to migrate across a Matrigel-coated membrane in an invasion chamber was evaluated. Following a $20-\mathrm{h}$ incubation period at $37^{\circ} \mathrm{C}$, the number of cells that had migrated across the membrane was determined. Invasive cells were stained, captured and counted under a microscope. (A and B) A representative result from three independent experiments with similar results. In the pGCsi-nm23-H1 group, the mean \pm SD was $134.8 \pm 6.66$, while in the liposome control group, this value was $54.4 \pm 3.42(\mathrm{P}<0.05)$. (A) Liposome group and (B) pGCsi-nm23-H1 group (x40). SD, standard deviation. the pGCsi-nm23-H1 K562 cells was examined in vitro. The cells migrated through a Matrigel-coated membrane during a 20 - $\mathrm{h}$ incubation period. The results revealed that the number of the pGCsi-nm23-H1 K562 cells that migrated into the lower compartment of the invasion chamber was markedly increased compared with the number of the liposome control K562 cells. Fig. 6 shows the mean \pm standard deviation (SD) of three independent experiments (pGCsi-nm23-H1 group, 112.4 \pm 4.56 ; and control group, 68.4 \pm 2.40 ).

\section{Discussion}

CML is a clonal myeloproliferative disorder that is characterized by the presence of the fusion oncogene, BCR-ABL. The constitutive expression of BCR-ABL leads to the unregulated production of mature myeloid cells in the bone marrow and their subsequent release into the blood (9). If untreated, CML will progress from a chronic to an accelerated phase over a number of years, prior to quickly proceeding to a terminal blast crisis phase, reminiscent of acute leukemia (10). The advent of tyrosine kinase inhibitors has led to an improved management of the disease. However, these drugs do not provide a cure as they are unable to eradicate the most primitive, quiescent fraction of CML stem cells (11).

The nm23-H1 gene is a metastatic suppressor that was identified in a melanoma cell line and is expressed in various tumors where their levels of expression are associated with a reduced or increased metastatic potential. $\mathrm{nm} 23-\mathrm{H} 1$ is one of $>20$ metastasis suppressor genes (MSGs) that have been confirmed in vivo. The gene is highly conserved from yeast to humans, implying a critical developmental function. Cell surface nm23-H1 has been previously observed in non-Hodgkin lymphoma (NHL) cells $(12,13)$ and certain myeloid cell lines $(14,15)$. Specific studies $(13,14)$ have demonstrated that tumors with a reduced expression of the $\mathrm{nm} 23$ gene are more prone to metastasis. It has been also previously documented that the expression of $\mathrm{nm} 23-\mathrm{H} 1$ transcripts and, more so, the levels of nm23-H1 protein in serum, provide strong indicators of prognosis, with higher values being associated with poorer overall survival (13-15).

The present study revealed a strong association between nm23-H1 gene expression and K562 cell survival in vitro. The MTT assay demonstrated that the stably-transfected pGCsi-nm23-H1 K562 cells exhibited a markedly increased ability to form colonies on soft agar. The number and sizes of the colonies that were formed by the pGCsi-nm23-H1 K562 cells were significantly increased compared with those of the liposome control group. Furthermore, to test whether the $\mathrm{nm} 23-\mathrm{H} 1$ gene was involved in the migration of the K562 cells, the effect of the invasiveness of the pGCsi-nm23-H1 K562 cells was examined in vitro. The results revealed that the number of the pGCsi-nm23-H1 K562 cells that migrated into the lower compartment of the invasion chamber was markedly increased compared with the liposome control K562 cells. This suggests that the behavior of the nm 23-H1 gene affects the biology of the CML cell lines, including growth, proliferation and invasiveness (16). These observations are consistent with other studies of solid tumors (17-19). However, the data from a study by Okabe-Kado et al (20) strongly indicated that the $\mathrm{nm} 23-\mathrm{H} 1$ gene may act as a tumor-derived survival factor in acute myeloid leukemia 
(AML). However, the study was unable to delineate between nm23-H1-binding AMLs and normal AMLs, in which the mechanism is likely to be active (20).

The experimental results from the present study suggest that the nm23-H1 gene is closely associated with the inhibition of metastasis. To assess the ultimate therapeutic potential of peptide vaccines derived from $\mathrm{nm} 23$, it will be necessary to determine, firstly, whether or not aberrant nm23-H1 expression is a widespread feature of CMLs and, secondly, whether the protein generates peptides that are able to act as functional antigens in HLA backgrounds other than HLA-A32. Given the widespread involvement of $\mathrm{nm} 23$ proteins in tumorigenesis, it will also be noteworthy to investigate the potential relevance of $\mathrm{nm} 23-\mathrm{H} 2$ as a therapeutic target in other cancers. The regulatory interdependence of $\mathrm{nm} 23-\mathrm{H} 2$ and c-myc provides a basis from which to design specific studies to elucidate the function of nm 23 proteins in normal and leukemic cells, which may contribute to our understanding of the molecular mechanisms underlying the development and progression of CML (21). Future studies should investigate the association between nm23-H1 binding and responses to CML therapies, and aim to determine the nature of the $\mathrm{nm} 23-\mathrm{H} 1$ receptor in CML, which may provide a novel target for adjunctive therapies.

\section{Acknowledgements}

This study was supported by a grant from the Shanghai Bureau of Health, China (no. 2010052).

\section{References}

1. Chang HJ, Yoo BC, Kim SW, et al: Significance of PML and p53 protein as molecular prognostic markers of gallbladder carcinomas. Pathol Oncol Res 13: 326-335, 2007.

2. Jiang WX, Song BG and Wang PJ: Expression of nm23, KAI1 and spiral computed tomography findings in primary gallbladder carcinoma. Chin Med J (Engl) 122: 2666-2668, 2009.

3. Marshall JC, Collins JW, Nakayama J, et al: Effect of inhibition of the lysophosphatidic acid receptor 1 on metastasis and metastatic dormancy in breast cancer. J Natl Cancer Inst 104: $1306-1319,2012$.

4. Boissan M, De Wever O, Lizarraga F, et al: Implication of metastasis suppressor NM23-H1 in maintaining adherens junctions and limiting the invasive potential of human cancer cells. Cancer Res 70: 7710-7722, 2010.

5. Liu HK, Wang Q, Li Y, et al: Inhibitory effects of gamma-tocotrienol on invasion and metastasis of human gastric adenocarcinoma SGC-7901 cells. J Nutr Biochem 21: 206-213, 2010.
6. Niitsu N, Hayama M, Yoshino T, et al: Multicentre phase II study of the CyclOBEAP regimen for patients with peripheral T-cell lymphoma with analysis of biomarkers. Br J Haematol 153: 582-588, 2011.

7. Niitsu N, Nakamine H, Okamoto M, et al; Adult Lymphoma Treatment Study Group, ALTSG: Expression of nm23-H1 is associated with poor prognosis in peripheral $\mathrm{T}$-cell lymphoma. Br J Haematol 123: 621-630, 2003.

8. Magyarosy E, Sebestyén A and Timár J: Expression of metastasis associated proteins, $\mathrm{CD} 44 \mathrm{v} 6$ and $\mathrm{nm} 23-\mathrm{H} 1$, in pediatric acute lymphoblastic leukemia. Anticancer Res 21: 819-823, 2001.

9. Bozkurt S, Uz B, Buyukasik Y, et al: Prognostic importance of additional cytogenetic anomalies in chronic myeloid leukemia. Med Oncol 30: 443, 2013.

10. Piccaluga PP, Paolini S, Bertuzzi C, et al: First-line treatment of chronic myeloid leukemia with nilotinib: critical evaluation. J Blood Med 3: 151-156, 2012.

11. Sawyers CL: The 2011 Gordon Wilson lecture: overcoming resistance to targeted cancer drugs. Trans Am Clin Climatol Assoc 123: 114-125, 2012.

12. Bircan S, Inamdar KV, Rassidakis GZ and Medeiros LJ: $\mathrm{nm} 23-\mathrm{H} 1$ expression in non-Hodgkin and Hodgkin lymphomas. Appl Immunohistochem Mol Morphol 16: 207-214, 2008.

13. Niitsu N, Honma Y, Iijima K, et al: Clinical significance of $\mathrm{nm} 23-\mathrm{H} 1$ proteins expressed on cell surface in non-Hodgkin's lymphoma. Leukemia 17: 196-202, 2003.

14. Lilly AJ, Khanim FL, Hayden RE, et al: Nm23-H1 indirectly promotes the survival of acute myeloid leukemia blast cells by binding to more mature components of the leukemic clone. Cancer Res 71: 1177-1186, 2011.

15. Bach E, Krahl R, Lange T, et al: Delayed processing of bone marrow samples reveals a prognostic pattern of NME mRNA expression in cytogenetically normal acute myeloid leukemia. Leuk Lymphoma 53: 1561-1568, 2012.

16. Jin L, Liu G, Zhang CH, et al: Nm23-H1 regulates the proliferation and differentiation of the human chronic myeloid leukemia K562 cell line: a functional proteomics study. Life Sci 84: 458-467, 2009.

17. Boissan M and Lacombe ML: NM23, an example of a metastasis suppressor gene. Bull Cancer 99: 431-440, 2012 (In French).

18. Conery AR, Sever S and Harlow E: Nucleoside diphosphate kinase Nm23-H1 regulates chromosomal stability by activating the GTPase dynamin during cytokinesis. Proc Natl Acad Sci USA 107: 15461-15466, 2010.

19. Zhao XS, Song PL, Sun B, et al: Arsenic trioxide inhibits metastatic potential of mouse hepatoma $\mathrm{H} 22$ cells in vitro and in vivo. Hepatobiliary Pancreat Dis Int 8: 510-517, 2009.

20. Okabe-Kado J, Kasukabe T, Honma Y, et al: Extracellular NM23-H1 protein inhibits the survival of primary cultured normal human peripheral blood mononuclear cells and activates the cytokine production. Int J Hematol 90: 143-152, 2009.

21. Tschiedel S, Gentilini C, Lange T, et al: Identification of NM23-H2 as a tumour-associated antigen in chronic myeloid leukaemia. Leukemia 22: 1542-1550, 2008. 